\title{
Educators as high-performance individuals and teams during a crisis
}

\author{
Christopher J. Lortie 1, Amy Lareau 2, and Andy Walshe 2 \\ 1. Department of Biology, York University, Toronto, ON, Canada. M3J1P3. \\ email \\ PH: 416.736.2100 FAX: 4167365696 \\ 2. Liminal Collective, LA, California, USA \\ email
}

Running title High-performance teaching during pandemic

Keywords bibliometrics, COVID-19 pandemic, education, responses, synthesis, teaching

Synopsis Educators and teachers are often front-line responders with children and families in a crisis. The COVID-19 pandemic was no exception. An effect-response model with adaptation can be applied to this multi-role challenge that educators faced to educate, protect, connect, and foster the well-being mentally and otherwise for students. This evidence can be used to explore the hypothesis that educators are high-performers and functionally elevate their performance under duress to serve the public. The relative importance of teacher performance during the COVID-19 pandemic was examined using scientific synthesis tools. An effect-response-adaptation model was used to model all research, and the relative importance of various responses implemented by teachers was explored. Research gaps and opportunities to better support and enable education in future are proposed. 


\section{Viewpoint}

A pandemic by definition has global global reach and thus dramatic ecosystem-level effects. The COVID-19 pandemic shifted every dimension of human endeavors including education. There was a demand and call for emergency remote teaching (1), and in many regions of the world, this process shifted from extemporaneous emergency education to persistent and sustained practice (2). At least 1.6 billion learners and $97 \%$ of the students globally were impacted (3). Educators and teachers are often front-line responders with children and families in a crisis (4). Teachers support and interact with children and their families. Schools define the local community and neighborhoods. Universities and colleges can define the character, culture, and economy of towns and cities. Thus, education is a fundamental component of human society $(5,6)$, and the performance and action of educators can dramatically influence societal good, function, and many other outcomes (7-9). The COVID-19 pandemic was no exception in its far-reaching effects as a crisis from individuals to communities, and educators were impacted and forced to respond and adapt.

Human performance is defined in many domains of research. In the field of social sciences that directly examines this concept, performance is a behavioral construct that is associated with a an individual (or team) with outcomes driven by both personality and net cognitive abilities that are in turn dependent on context and task (10). This complexity is not superfluous. The traits and skills of an individual are the starting point or substrate for models of performance, and the cognitive abilities inform capacity to utilize these assets in meeting specified outcomes. These processes of leveraging traits and skills do not happen in isolation of context or from the challenge of a specific task. Consequently, routine performance in exceptionally challenging circumstances or for particularly demanding tasks is exceptional. This premise is fundamental to educators supporting learning and providing instruction throughout a pandemic. Stoicism or self-efficacy in spite of incredible adversity has been proposed as the foundation that defines human performance (11), and many other disciplines of performance research echo this sentiment (12-15). The wellbeing and performance of teachers is thus a critical form of human capital in education and society (16) and a potentially important metric of educational integrity (17). Furthermore, we propose that this resilience in crises is a critical lever in maintaining a functional society.

A model that supports this starting point for educator performance in crisis contexts is needed. An effectresponse model with long-term adaptation can be applied to this multi-role challenge that educators face to educate, protect, connect, and foster the well-being mentally and otherwise for students. We can borrow from the competition and stress theory in the environmental sciences to structure an analysis of education research here. Effects are external drivers on our focal subjects, i.e. educators, that can be contextual or from other subjects $(18,19)$. This can be more simply conceived of as stress $(20,21)$ - here from the pandemic and emergency-to-sustained remote teaching in addition to health and safety challenges to educators and students. Responses can include the strategies, solutions, and behaviors of individual educators to these stressors. Finally, adaptations are the potential population or society-level institutional strategies that collectively evolved throughout this crisis in schools or universities to address the stress and challenges of pandemic teaching at scale. This model can be applied to the contemporary and ground-breaking research in education to explore the hypothesis that educators are high-performers and functionally elevated their performance under duress to serve the public in the last 16-18 months. The implication of this approach is that we can learn from educational research right now to inform and develop more resilient and long-term strategies for both conventional and remote teaching in future.

The extent of published, peer-reviewed research associated with the COID-19 pandemic is nothing short of staggering. Conservative queries for key terms in Google Scholar and The Web of Science returned over 200,000 papers from the start of 2020 to now (explored June 2021). This does not include archived preprints. It has been estimated that 137 papers per day were published on COVID-19 since February 2020 (22). Productivity rates in academia vary based on discipline and topic specificity, but this volume and rate of research publication is likely an order of magnitude greater than precedents for most if not all other previous human endeavors shared in journals $(23,24)$. Terms directly associated with teaching and education were scoped, and at least 2154 papers have been published (Table 1). In academic research, it is not uncommon for scientific synthesis papers such as reviews, systematic-reviews, and meta-analyses to aggregate and compile findings from individual, primary studies to explore larger, consistent patterns in research when sufficient evidence has accumulated $(25,26)$. More research is not necessarily ideal or critical for discovery, but this 
corpus of work does provide a needed opportunity to examine the responses of educators to the pandemic through scientific synthesis. Introspection of collective work at key points can also lead to novel directions of research and identify gaps.

\section{Review of syntheses}

A set of queries were first scoped using The Web of Science bibliometrics engine to explore and aggregate the peer-reviewed, indexed research from 2020 to June 2021 on education and the pandemic (27). The number of meta-analyses, reviews, and systematic reviews totaled 99 instances (Table 1). These synthesis, higher-order studies were then reviewed for relevance to education and responses to the COVID-19 pandemic, and all remaining studies were classified using the effect-response-adaptation model proposed above. Adaptation or institutional and organization-level strategies comprised the least significantly synthesized form of COVID-19 pandemic education research followed by pandemic effects on educators (Figure 1). The response of educators to the pandemic in their teaching was the most studied comprising $57 \%$ of all studies. A total of 7039 individuals were collectively summarized in these studies.

A more detailed analysis was applied to the response literature using the $\mathrm{R}$ programming language (28) on the text and tabular frequencies, and when reported, quantitative estimates. The relative frequency of teaching responses by individual educators were summarized (Figure 2). A total of 11 distinct generalized responses were reported in the synthesis literature, and the mean success rate describes the frequency that these interventions or strategies were implemented during the pandemic (Figure 2). Success was defined in terms of frequency of study and application by educators, i.e. the sign of positive and negative reciprocal effects on students was not summarized in these syntheses. These findings do strongly suggest that some strategies with relatively higher frequencies, i.e. arbitrarily greater than $50 \%$ of the time for instance, were amenable to future introspection, innovation, and potentially reflect efficacy estimates for emergency and remote instruction. In a perfunctory analysis to examine high-level trends, these relative occurrence data were used to calculate a weighted-effect size estimate using the escalc function in the metafor package in $\mathrm{R}$ (29). The proportion of strategies implemented by educators were thus converted to incidence rates weighted by the relative sample sizes, i.e. an intervention tried $50 \%$ of the time with a small class for a few students is not weighted equivalently to a study tried with several hundred individuals. The mean incidence effect sizes were effective and positive (Random-effects meta-analysis, Chi-square value $=1.69, \mathrm{p}=0.0001$ ) whilst the various strategies reported significantly differed from one another (Random-effects meta-analysis with strategy as moderator, $\mathrm{F}$ value $=2.86, \mathrm{p}=0.008$ ). Similar to the raw implementation success rate data, teaching strategy and instruction emerged as frequent response mechanisms used by educators during the pandemic.

\section{Implications}

The conceptual model of effect-response-adaptation successfully described and classified the corpus of peer-reviewed, indexed research literature published on education during the COVID-19 pandemic to date. Response by educators was frequently examined, and these findings strongly suggest that virtually every single component of remote, distributed learning and educator performance expressed a deep and meaningful change. In particular, the human factors in this crisis such as teaching strategies, instruction, and flexibility not the new technology nor novel technique strategies - were the most fluent and significantly diverged from the previous educational norms suggesting a critical research gap in supporting e-learning and educators in future can be better enabled by developments in these specific fields. Professional development, mindfulness training, and efficacy also emerged as salient issues for innovation in the future of performance for educators and institutions or schools that support instruction. A recent survey published responses of $\sim 6000$ teachers in the USA strongly suggested that their sense of efficacy at the tail of the pandemic was significantly eroded (30). This evidence underscores the importance of population, societal, and institutional-level adaptations now desperately needed to reduce impacts on the sustained performance of educators. Individual responses and routine teaching in adverse conditions, i.e. exceptional performance, can only get us so far, and as a society, we must globally consider deep and novel investments in this form of human capital. Education is a pillar of society that buttresses our collective wellbeing, and attention to the efficacy of educators as responders to change in all contexts will provide resilience in remote and many other future teaching contexts. 


\section{Literature cited}

1. S. Abaci, J. Robertson, H. Linklater, F. McNeill, Educational Technology Research and Development 69, $29(2021 / 02 / 01,2021)$.

2. M. Usher, A. Hershkovitz, A. Forkosh-Baruch, British Journal of Educational Technology n/a, (2021/05/16, 2021).

3. S. Pokhrel, R. Chhetri, Higher Education for the Future 8, 133 (2021/01/01, 2021).

4. L. Evans, J. Oehler-Stinnett, School Psychology International 27, 33 (2006).

5. A. Javed, Res Publica 8, 150 (2003).

6. F. Idris, Z. Hassan, A. Ya'acob, S. K. Gill, N. A. M. Awal, Procedia - Social and Behavioral Sciences $59,443(2012 / 10 / 17 /, 2012)$.

7. D. E. Campbell, MEASURING THE EFFECTS OF EDUCATION ON HEALTH AND CIVIC ENGAGEMENT: PROCEEDINGS OF THE COPENHAGEN SYMPOSIUM 3, 25 (2006).

8. L. Darling-Hammond, L. Flook, C. Cook-Harvey, B. Barron, D. Osher, Applied Developmental Science 24, $97(2020 / 04 / 02,2020)$.

9. A. Bacher-Hicks, J. Goodman, C. Mulhern, Journal of Public Economics 193, 104345 (2021/01/01/, 2021).

10. S. J. Motowildo, W. C. Borman, M. J. Schmit, Human Performance 10, 71 (1997/06/01, 1997).

11. T. O. Peterson, R. B. Arnn, Performance Improvement Quarterly 18, 5 (2008).

12. M. Neelen, P. A. Kirschner, Evidence-informed learning design: creating training to improve performance. (Kogan Page, London, 2020).

13. R. Samra, The British Journal of Psychiatry 216, 294 (2020).

14. F. J. Garrigos Simon, E. Pagain, J. S. Garcia, Esic Market Economics and Business Journal 52, 19 (2021).

15. Y. Xuqun, Social Sciences in China 42, 135 (2021/01/02, 2021).

16. Ş. Zlate, C. Enache, Procedia - Social and Behavioral Sciences 180, 136 (2015/05/05/, 2015).

17. K. S. Hranchuk, M. J. Williams, Behavioral Interventions 36, 355 (2021/04/01, 2021).

18. D. E. Goldberg, R. Turkington, L. Olsvig-Whittaker, Folia Geobotanica Phytotax 30, 231 (1995).

19. D. E. Goldberg, T. Rajaniemi, J. Gurevitch, A. Stewart-Oaten, Ecology 80, 1118 (1999).

20. A. F. Sanders, Acta Psychologica 53, 61 (1983/04/01/, 1983).

21. C. J. Lortie, in Positive Plant Interactions and Community Dynamics., F. I. Pugnaire, Ed. (CRC Press, Boca Raton, FL, 2010), pp. 59-78.

22. N. S. L. Yeo-Teh, B. L. Tang, Accountability in Research 28, 47 (2021/01/02, 2021).

23. P. O. Larsen, M. von Ins, Scientometrics 84, 575 (2010/09/01, 2010).

24. E. Z. Cameron, M. E. Gray, A. M. White, Trends in Ecology \& Evolution 28, 7 (2013/01/01/, 2013).

25. C. J. Lortie, Oikos 123, 897 (2014).

26. B. S. Halpern et al., BioScience, (2020).

27. C. Analytics, Web of Science Core Collection Accessed July 2020, (2020).

28. R-Development-Core-Team, R: a language and environment for statistical computing., (R foundation for Statistical Computing, Vienna, Austria, 2021), vol. 4.0.5.

29. W. Viechtbauer, CRAN, Version 2.0 (2017).

30. M. A. Kraft, N. S. Simon, M. A. Lyon, EdWorkingPapers.com, (August 2020, 2020). 
Table 1. A list of search returns examining research on teaching and the COVID-19 pandemic. Queries were in done in May 2021 and were from 2020 to present using the bibliometrics tool The Web of Science.

\begin{tabular}{rlrlll}
\hline item & search & returns & refine & years & usefulness \\
\hline 1 & pandemic \& teaching & 2154 & topic & $2020-21$ & medium \\
2 & pandemic \& teacher & 259 & topic*title & $2020-21$ & medium \\
3 & pandemic \& teach* & 851 & topic*title & $2020-21$ & high \\
4 & covid \& teach* $_{5}$ & 1019 & topic*title & $2020-21$ & high \\
5 & covid \& teach* \& stress & 56 & topictitletopic & $2020-21$ & very high \\
6 & covid \& teach* \& response & 167 & topictitletopic & $2020-21$ & very high \\
7 & covid \& teach* \& effect $_{8}$ & 114 & topictitletopic & $2020-21$ & very high \\
8 & covid \& teach* \& meta* & 14 & topictitletopic & $2020-21$ & very high \\
9 & covid \& teach* \& review* $_{10}$ & 66 & topictitletopic & $2020-21$ & very high \\
& covid \& teach* \& systematic* & 19 & topictitletopic & $2020-21$ & very high \\
\hline
\end{tabular}


Figure 1. The relative frequency of studies during the COVID-19 pandemic on education classified by effect-response-adaptation (categories described in text). These data from number of publications queried from The Web of Science in June 2021.

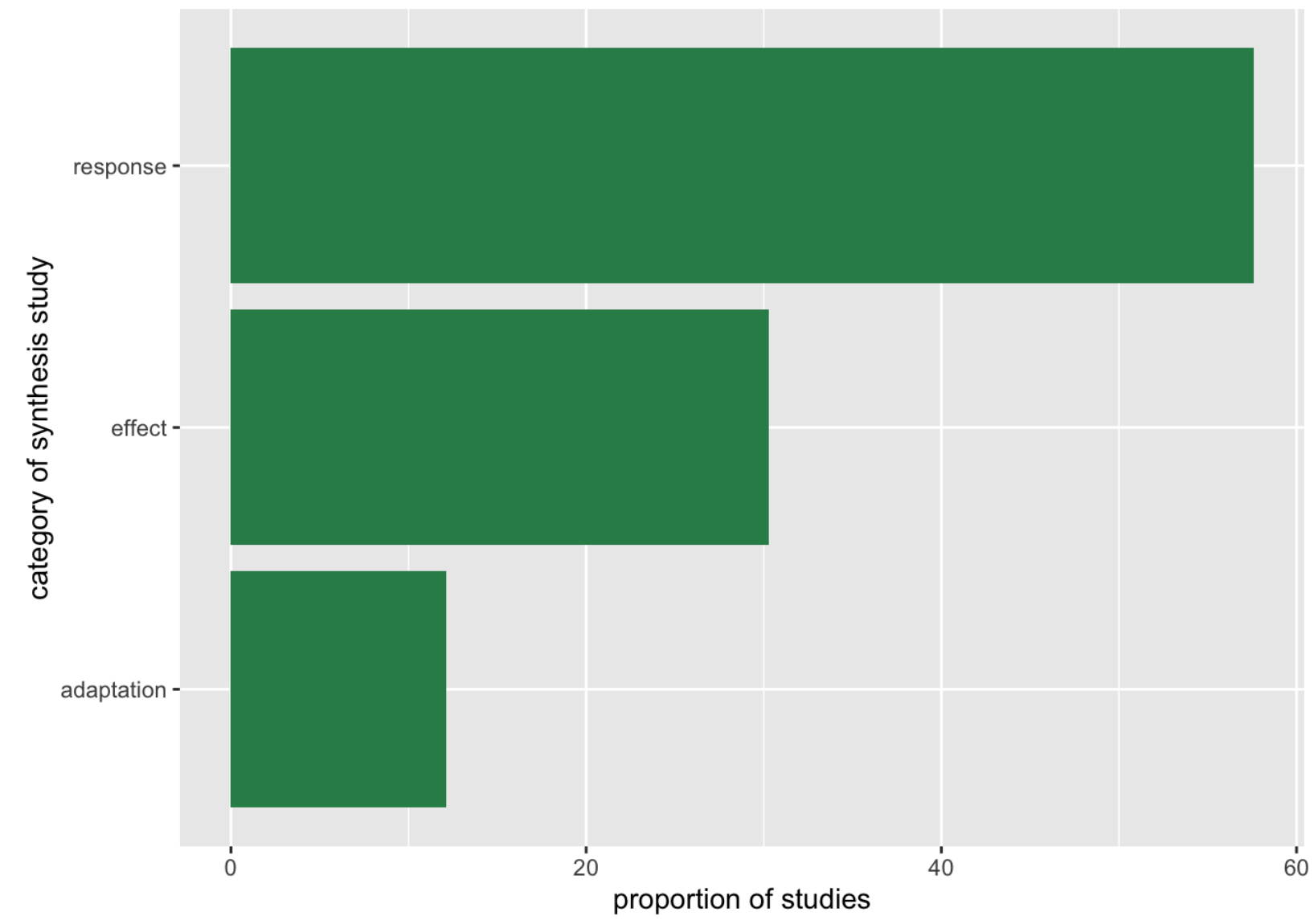


Figure 2. The proportion of responses reported by educators to the COVID-19 pandemic estimated from the synthesis studies returned from the Web of Science (queried June 2021). A total of 7039 individuals were studied in this context with respect to the interventions and strategies implemented by educators in emergency remote teaching and sustained practice as distributed teaching persisted.

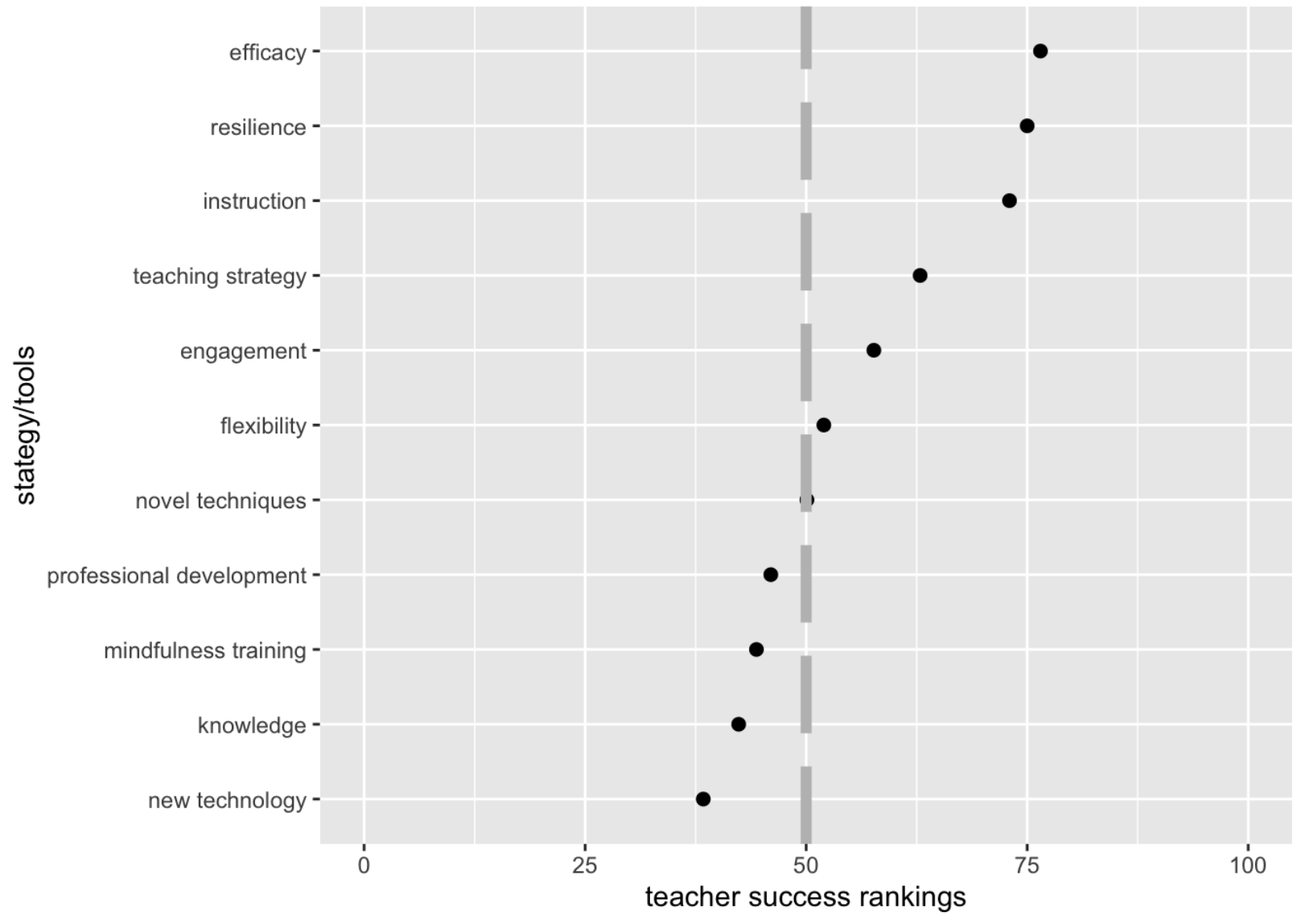


Figure 3. The mean effect size measures for incidence rates used in a meta-analysis of strategies used by educators during the COVID-19 pandemic. The relative frequency implemented was weighted by the sample size listed in each study (or in number of studies summarized in synthesis studies on this topic from The Web of Science).

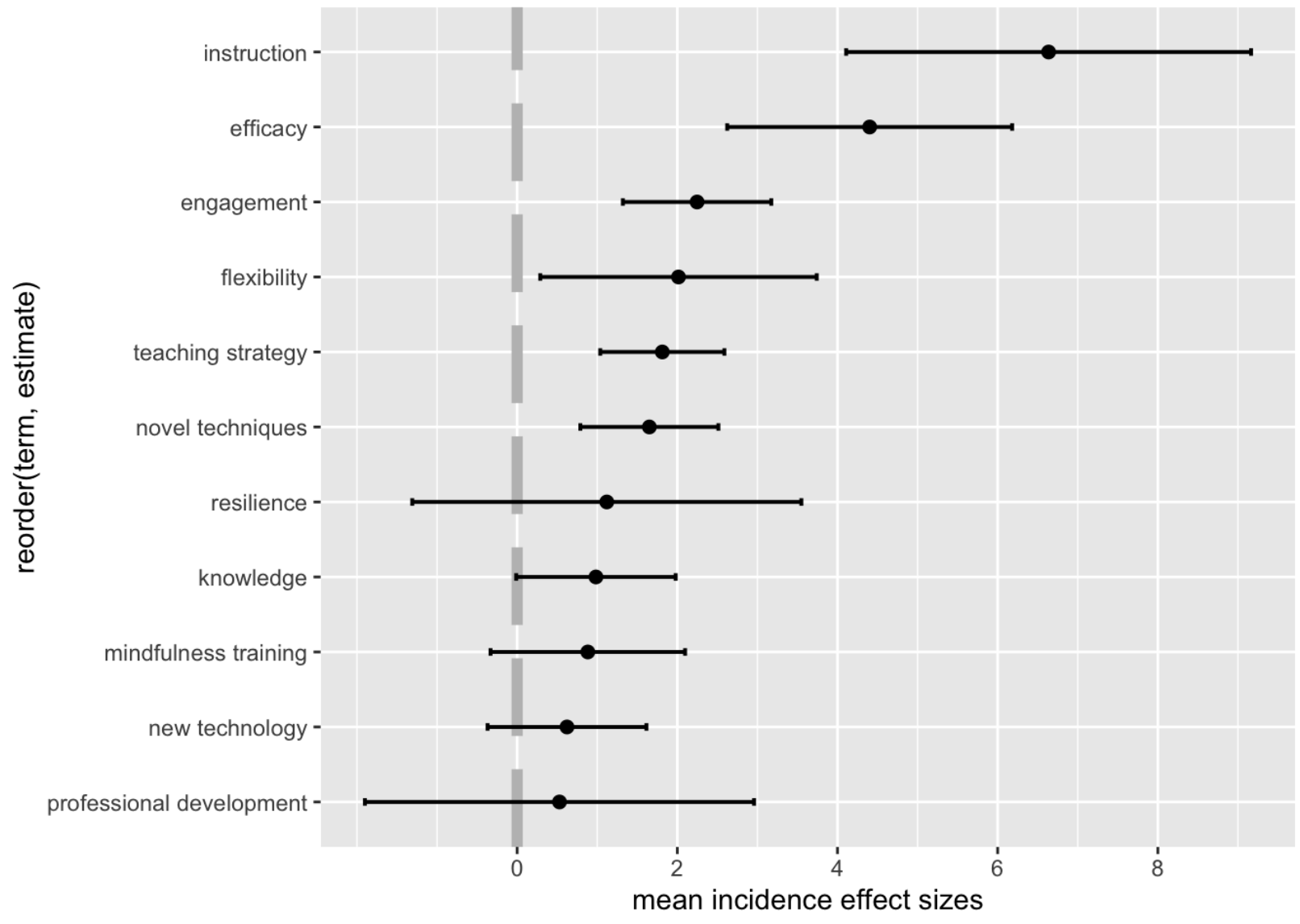

